\title{
A Lotus 1-2-3-based system for recording and maintaining body weight of laboratory animals
}

\author{
JOSEPH S. ALI \\ U.S. Environmental Protection Agency, Research Triangle Park, North Carolina \\ VALERIE B. OLSZYK, DUSTY D. DUNN, KEH-LIANG A. LEE, and SHARON M. KENDALL \\ ManTech Environmental Technology, Inc., Research Triangle Park, North Carolina \\ and \\ RICHARD R. RHODERICK and PHILIP J. BUSHNELL \\ U.S. Environmental Protection Agency, Research Triangle Park, North Carolina
}

\begin{abstract}
Body-weight maintenance is required in a variety of behavioral and physiological studies. A PC-based animal-weighing system is described; it features automated data collection and allows for accurate control of body weight in test animals via manipulation of food intake. Major system components are a PC XT-compatible computer, Lotus 1-2-3 and National Instruments Measure software, and a digital electronic balance. The system is controlled by Lotus macros, which present menus to the user. Menus provide for the basic operations of the system, including establishing new data files, collecting weight data, printing, and transferring files to a laboratory minicomputer. The optional weight-maintenance algorithm calculates the amount of food necessary to maintain target body weights. Some important aspects of this algorithm, including maintenance-feeding and gain parameters, can be manipulated to reduce weight oscillation in test animals or to accommodate the maintenance of body weight of different animal species. The system facilitates acquisition and recording of weight data, minimizes the frequency of error, and reduces variability both within and across animals when body-weight maintenance is required.
\end{abstract}

The weight of a test animal is a basic measure of health and vitality, widely used in toxicology and pharmacology to monitor the effects of chemical or physical treatments (e.g., Gad, 1982; Moser, McCormick, Creason, \& MacPhail, 1988; Zbinden, 1988). In addition, many studies of behavior rely upon appetite to motivate animals to perform tasks of interest (Hineline, 1986; Kaplan et al., 1959). Control of appetite typically involves control of feeding, which is often based upon maintaining an animal at a target body weight (Hurwitz \& Davis, 1983); conversely, body weight may be manipulated experimentally to explore the effects of nutritional status on health and longevity (Holehan \& Merry, 1986; Yu, Masoro, Murata, Bertrand, \& Lynd, 1982).

\footnotetext{
The research described in this article has been reviewed by the Health Effects Research Laboratory, U.S. Environmental Protection Agency, and approved for publication. Approval does not signify that the contents necessarily reflect the views and policies of the Agency nor does mention of trade names or commercial products constitute endorsement or recommendation for use. The authors acknowledge the invaluable assistance of Kaye Rigsbee of PRI, Inc., for her help in testing the system during its development. We also thank Ned Collins of PRI, Inc., and Richard Linko of the EPA for assistance in coordinating the system's use in the laboratory and Ezra Berman and Christopher Gordon for helpful reviews of the manuscript. Correspondence may be addressed to Joseph S. Ali, Neurotoxicology Division, MD 74B, U.S. Environmental Protection Agency, Research Triangle Park, NC 27711.
}

Automated acquisition of body weights of experimental animals greatly facilitates collection of the data necessary for this purpose. In the case of body-weight maintenance, the additional problem of estimating the food required to keep an animal at a target weight can be eased by means of an algorithm for calculating the amount of food needed for the present feeding, based upon knowledge of a target weight, the present weight of the animal, and an estimate of the animal's daily caloric need. A similar method has been devised to maintain horses' dietary needs, which vary depending upon developmental stage and exercise requirements (Pagan, 1989).

The present animal-weighing system was designed and implemented to improve the acquisition and processing of data collected during daily weighing operations performed in a small animal colony used for a research program investigating the health effects of environmental chemicals. An additional goal was to develop an animal-feeding algorithm that would allow an animal's weight to be maintained at a prescribed target weight. Prior to the development of this system, the animals' weights were manually tabulated and entered into a computer data file for analysis. Errors could occur when the animal weights were recorded and when they were entered into a data file. The animal weights were maintained at target values by a subjective algorithm applied intuitively by experienced animal caretakers. 
The present system eliminates manual handling of animal weights; it provides a printed copy of a "weighing worksheet" that contains session-identification information and a tabulated listing of the animal-identification numbers, weights, and, optionally, the amount of food to feed each animal to achieve target weights.

\section{SYSTEM OVERVIEW}

The system consists of a PC XT computer running a Lotus 1-2-3 spreadsheet under macro program control. System components are: an 8-MHz PC XT computer with $640 \mathrm{~K}$ of RAM, dual $360 \mathrm{~K}, 5.25$-in. drives, a $20 \mathrm{MB}$ hard disk card, two RS-232 serial ports, a parallel printer port, and a monochrome monitor; a dot-matrix printer; Lotus 1-2-3 spreadsheet software; National Instruments Measure (formerly Lotus Measure), a collection of data acquisition software drivers for Lotus 1-2-3; and a Mettler Model PL3000 balance with a BCD to current loop interface (Model CL 240) and a current loop to RS-232 interface (Model CL 249). Newer balances that have a built-in RS-232 interface would not require the last two items. The RS-232 output of the balance is connected to one serial port (COM 1), and the second serial port is used to transfer weight data to laboratory minicomputers. A single floppy-disk drive is required to load the application software to the hard disk.

The cost to implement a weighing system, excluding the cost of the PC itself, is about $\$ 1,980.00$ at today's prices, including Lotus 1-2-3 Version 2.2, National Instruments Measure, and an electronic balance with an RS-232 data interface. Many laboratories already have Lotus 1-2-3, and many existing balances can be retrofitted with an RS-232 data interface. In this case, the cost would drop to about $\$ 650.00$. The selected balance should either have a variable-integration-time feature or an animal-weighing mode to accommodate animal motion. The transmission parameters of the balance's data interface are unimportant because the Measure software can be configured to accommodate all commonly used parameter values.

The software is menu-driven. The user selects functions to perform from a control panel in which the menu choices are displayed exactly as are the menu choices in Lotus itself. The menu choices presented are not Lotus commands, however; instead, they are functions that we have defined for the application at hand. The functions available are: (1) enter a series of animal-identification numbers and, optionally, animal target weights; (2) retrieve a worksheet that has been preloaded with animal-identification numbers and collect weight data; (3) print a worksheet that is currently displayed on the monitor; (4) display a list of file names that contain the animal-identification numbers; and (5) quit the session and exit Lotus 1-2-3.

The program has several error-trapping routines that prevent the macro code from terminating when an error is encountered. This feature is important since it permits the system to be used by animal-care personnel, who typi- cally are not trained Lotus software users. For example, if a user attempts to print a worksheet with the printer in the off-line mode, error-trapping routines give the user a chance to correct the problem detected by Lotus 1-2-3 before proceeding. (Under normal macro operation, the user is returned to the Lotus READY mode, at which point the software is no longer menu-driven and Lotus 1-2-3 simply waits for a command from the user.) Error detection presents a special error screen to the user. In addition to an error message, this screen provides instructions to the user on how to correct the problem. Continuing with the example above, if the printer were off-line, the error-message screen would indicate this condition and prompt the user to put the printer in the on-line (ready) mode. The printing process is then initiated.

The software also protects certain areas of the worksheet. The user is given limited cursor-movement capability to edit entered data; however, if the user attempts to change or overwrite a protected area of the worksheet, an error screen is presented and the user is prompted to move out of the protected area. This prevents the user from inadvertently deleting or changing the worksheet template or the macro code stored in an area of the spreadsheet out of the view of the user.

The software (macro code) is contained in a worksheet file called AUTO123. WK1. Lotus users will recognize this as the standard name for the worksheet that is automatically loaded when Lotus 1-2-3 is run. Furthermore, the macro code in the worksheet is named in such a way that it automatically executes when the spreadsheet is loaded; therefore, the user is under macro control at all times. As a result, the user does not need significant expertise with Lotus 1-2-3 commands. After start-up, the main menu described below is displayed.

\section{DESCRIPTION OF MAJOR FUNCTIONS IN MAIN MENU}

Figure 1 shows the main menu with the menu choices on the control panel at the top of the page. The menu choice COLLECT_DATA is shown in bold type. The major functions are described briefly below in the order in which they would normally be used.

1. Load animal-identification numbers. Before any weights can be collected, the user prepares a worksheet that contains experiment-identification information as well as a set of animal-identification numbers. The user has the option of entering a target weight for each animal. This worksheet is prepared only once for each experiment. The worksheet is recalled and reused each time weights are to be collected for that experiment. The program requires that the animals be weighed in the order in which their identification numbers were entered. The program is designed to accept up to 200 animal-identification numbers per experiment.

After the LOAD_ID NO'S function is selected, the program prompts the user to select the principal investigator (PI). Known users of the system can be included 


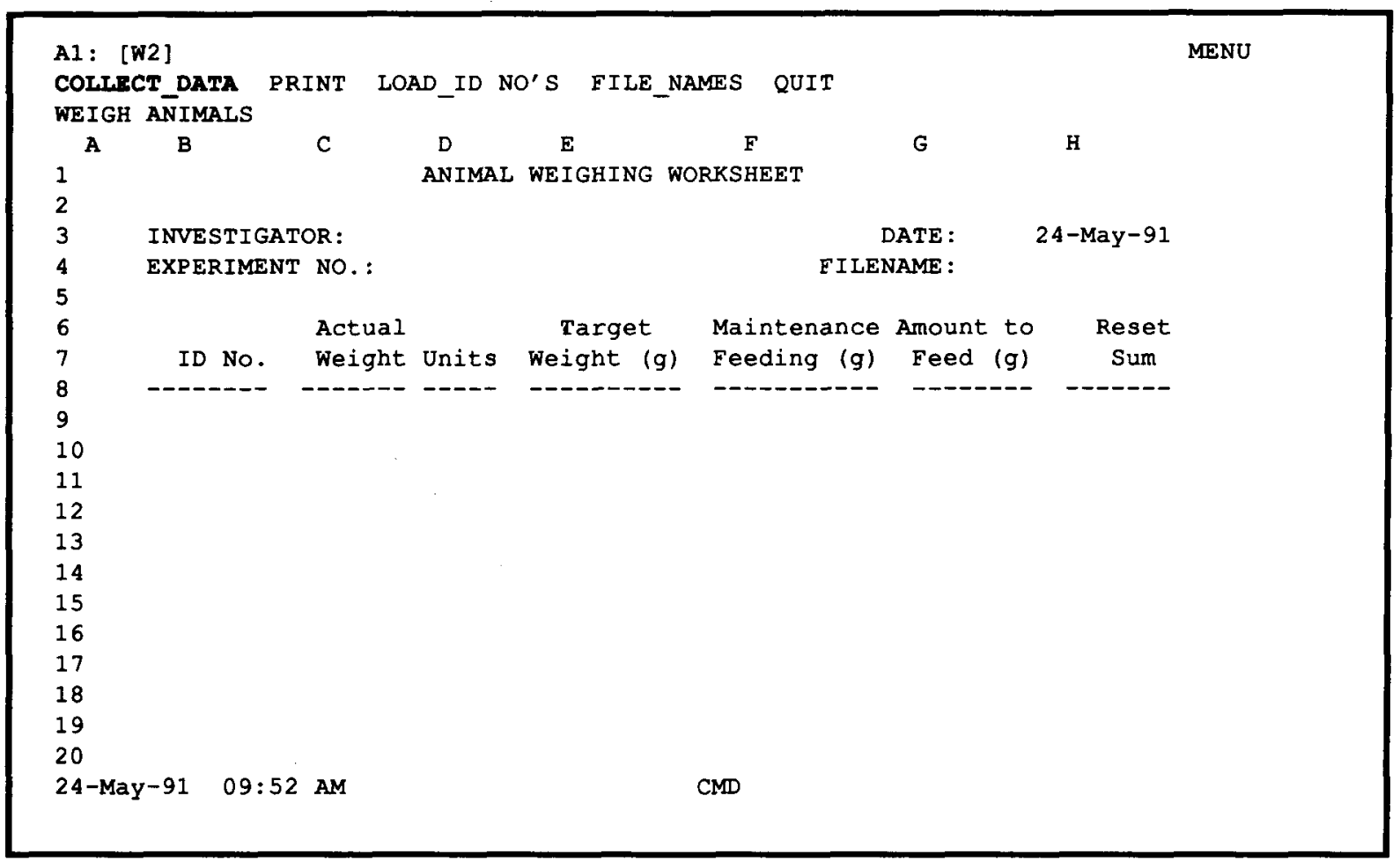

Figure 1. The main menu screen. The second line shows the menu choices; the third line has a short description of the bold-type choice on line two. The letters CMD on the last line indicate that the system is under macro program control.

in the program and are displayed on the control panel as a menu choice. New users are prompted to type in their names. Users are prompted for an experiment number.

Immediately after the experiment number is entered by the user, a file name is created; it consists of the first four characters of the PI's name followed by an underscore character and the three-character experiment number. For example, if the PI's name is Smith and the experiment number is G50, the file name will be SMIT_G50. This technique of naming files facilitates file identification.

Storing target weights is optional. If this option is chosen, the program prompts the user for the target weight along with the animal-identification number. After a target weight is entered, an initial value of the maintenance feeding and the reset sum (defined below) are automatically loaded into the worksheet. At the end of the data entry session, the program automatically saves the data as a Lotus worksheet to hard disk in a subdirectory called WEIGHTS and then returns to the main menu.

2. Collect animal weights. To proced with animal weighing, the menu choice COLLECT_DATA is selected. This menu choice will prompt the user to enter the PI name and the experiment number. The program will then recall the worksheet containing the animal numbers and prompt the user to weigh each animal in the data set. The operator then weighs an animal by placing it on the balance and pressing a footswitch. The switch indicates to the balance to transfer a reading to the computer when the balance determines that a preset level of stability (integration time $=1.2 \mathrm{sec}$, tolerance band set as wide as possible) has been achieved. If target weights were entered along with the animal-identification numbers when the worksheet was created, the program will compute the amount of food to feed each animal to the nearest gram after it has been weighed.

Editing capability has been provided in this function. If a measured weight is in doubt, it can be recollected by first positioning the cursor with the $U$ or $D$ key followed by ENTER and then reweighing the animal.

At the end of the weighing session, the user is given the opportunity to save the weights to disk. The file name will be the same as the worksheet name containing the animal numbers; however, the file-name extension will be PRN because the animal-weight files are saved as ASCII text files. For example, if the animal-identification number worksheet file name is SMIT_G50.WK1, then the animal-weights file name will be SMIT_G50.PRN, and it will be stored in a subdirectory called WEIGHTS.

3. Print the animal-weights worksheet. The worksheet is usually printed after loading the animal-identification numbers and after a weighing session. After performing either of these tasks, the user is returned to the main menu and the worksheet remains filled with the data just collected and is ready for printing. To print a worksheet, the user selects the PRINT menu choice. A sample printout is provided (Figure 2). 


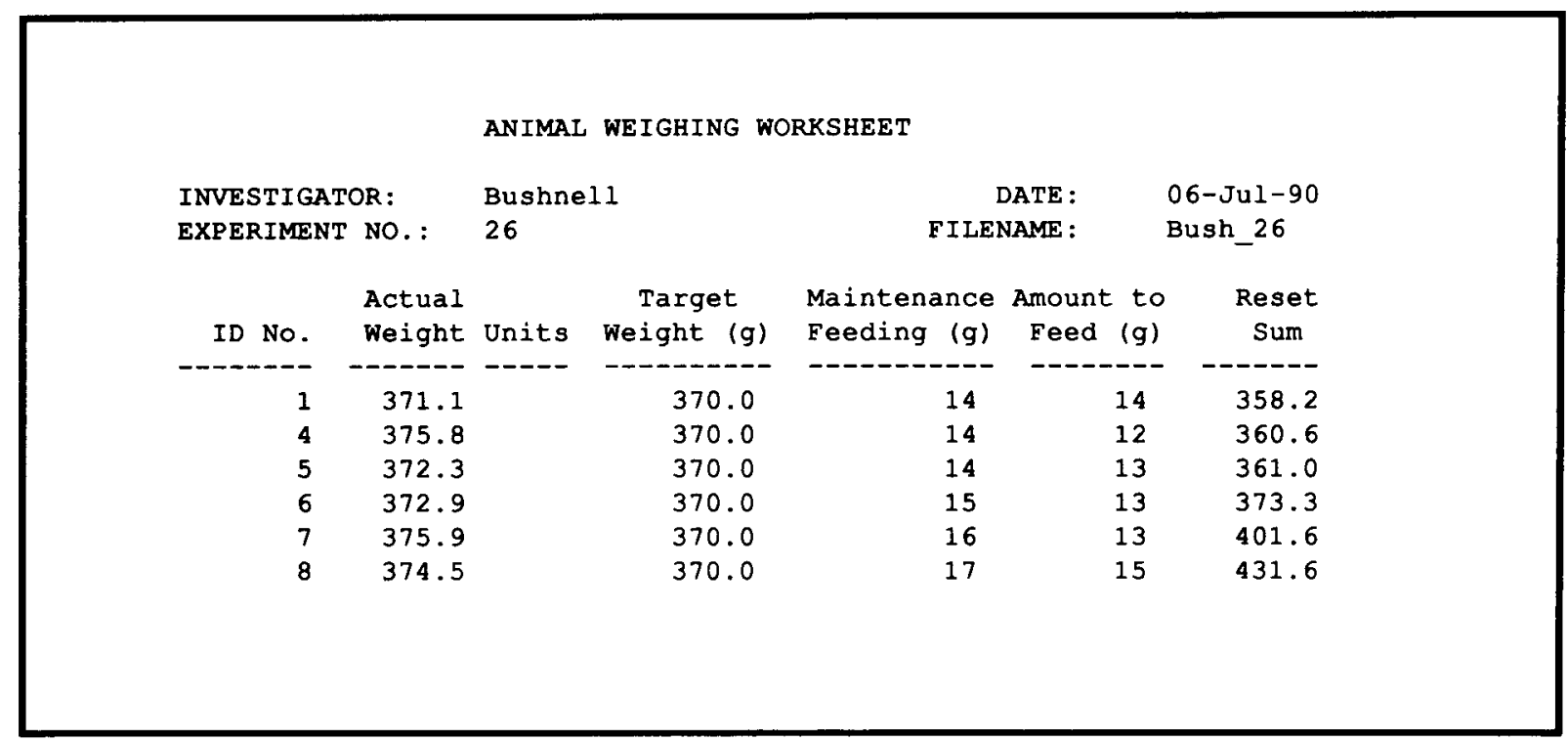

Figure 2. A sample printout of the animal-weighing worksheet with weight maintenance. The animals are fed the amounts shown in the second-to-last column. Had target weights not been entered when the animal-identification numbers were first loaded by the user, only columns 1 and 2 would contain values. The Units column is present for those balances that output this information. These data are available to the user on a daily basis and transferred to a central CPU for storage and further analysis as necessary.

4. Display a list of animal-identification file names. This function allows the user to display the file names of all the prepared worksheets containing the animal-identification numbers. Embedded in the file name is the experiment number assigned by the user. To use this function, the user simply selects the FILE_NAMES menu choice.

5. Quit the program. This menu choice is selected when the user is ready to exit the program.

\section{DATA-FILE MAINTENANCE}

After all groups of animals have been weighed, the WEIGHTS subdirectory will contain an ASCII data file (which can be identified by the file-name extension .PRN) for each group of animals weighed. Each of these files contains only the current day's data. Each day after all animals have been weighed and the animal caretaker has exited the weighing worksheet and Lotus 1-2-3, a small batch file (merge.bat) is called that merges each *.PRN file with a cumulative file with the same name, but with a .CUM extension. On the first day the system is run, the cumulative file is automatically created; on each subsequent day the system is run, the current day's data are appended to the corresponding cumulative file. For example, SMIT_G50.PRN is merged with another file called SMIT_G50.CUM, and the SMIT_G50.PRN file is deleted. About once a week all cumulative files are transferred into each investigator's account on our laboratory minicomputer via a network interface. After a successful file transfer, the cumulative file is deleted from the PC.

\section{WEIGHT-MAINTENANCE ALGORITHM}

Prior to the development of this system, maintaining an animal's body weight was done "by hand" by an experienced animal caretaker. We have developed an algorithm that computes the amount to feed an animal on a particular day. The amount of food to feed an animal to control body weight consists of two components: a maintenance-feeding component and a proportional corrective component to make up for short-term fluctuations in the animal's body weight. The maintenance feeding reflects the long-term daily caloric need of the animal. The maintenance feeding required to keep an animal at a certain body weight gradually diminishes with age. Therefore, if an algorithm is to control an animal's body weight successfully over a significant portion of an animal's life span, it must compensate for changes that occur in the maintenance-feeding requirement. Since changes in maintenance-feeding requirement occur gradually, it is reasonable to consider using a series summation term in a weight-maintenance algorithm. On the other hand, to compensate for short-term weight fluctuations, a term proportional to the daily weight deviation from the target weight would be suitable. The following algorithm was developed assuming that the animals are weighed daily, that the feeding is based on the results of that weighing, and that the animals have ad-lib access to water.

$$
\begin{aligned}
\mathrm{ATF}= & \text { reset gain } \times \sum_{j=1}^{i}\left(\mathrm{TW}-\mathrm{WT}_{j}\right) \\
& + \text { proportional gain } \times\left(\mathrm{TW}-\mathrm{WT}_{i}\right),
\end{aligned}
$$


where ATF is the amount of food to feed, rounded to the nearest gram, reset gain is a scale factor for the next term in the equation called the reset sum, proportional gain is the scale factor for the error term, the difference between the target weight, TW, and the current weight of the animal, $\mathrm{WT}_{i}$, and $i$ is the current day. The sum is taken across all days, except as noted below.

The product of the reset gain and the reset sum is the maintenance feeding. For the Long-Evans rats that we normally use, the range of maintenance feeding is expected to be between 5 and $30 \mathrm{~g}$. When animals are first entered into the system through the LOAD_ID NO'S function, the program loads the maintenance-feeding field with an initial value of $15 \mathrm{~g}$. The initial reset sum is derived from the initial maintenance feeding by dividing it by the reset gain. Thus, for an initial maintenance feeding of $15 \mathrm{~g}$ and a reset gain of 0.04 , the initial reset sum is $375 \mathrm{~g}$. This value is loaded into the reset-sum field of the worksheet. The reset gain and the proportional gain are fixed in the software at 0.04 and 0.5 , respectively. The value for reset gain was determined by computer modeling, while the proportional gain was determined empirically.

Before the ATF is reported to the user, it is limited by the following function:

$$
0 \leq \mathrm{ATF} \leq 30 \mathrm{~g} \text {. }
$$

That is, if the current body weight were so high that Equation 1 produced a negative result, then $0 \mathrm{~g}$ would be reported as the amount to feed; likewise, if the body weight were so low that Equation 1 produced a result greater than $30 \mathrm{~g}$, then $30 \mathrm{~g}$ would be reported as the amount to feed. Furthermore, the reset-sum term is limited in that it is updated only when:

$$
\left|\mathrm{TW}-\mathrm{WT}_{i}\right|<20 \mathrm{~g} .
$$

It should be noted here that when this limit condition is applied, some members of the summation series of Equation 1 will be missing. That is, the reset sum is actually the summation of target-weight minus current-weight terms for those terms with an absolute difference of less than $20 \mathrm{~g}$.

This limit is imposed on the reset sum to prevent what is called "reset windup" in control systems terminology. Reset windup occurs in control systems employing integral or summation terms when a large difference between the controlled variable, the weight of the animal, and the setpoint, the target weight, persists for a long time. This would occur in our system when a young animal arrives in the animal colony and is placed on the weight-maintenance algorithm with a current weight much less than the target weight. If there were no limit imposed on the reset sum, this term would become large and cause a large overshoot in the body weight as the animal approached the target weight. By waiting until the animal's weight is within $20 \mathrm{~g}$ of setpoint before updating the reset-sum term, reset windup, and hence the weight overshoot, is minimized.

Since the reset sum is a summation of previous error terms, it must be stored and updated in the animal- identification worksheet each day that the animals are weighed. There is the potential for a problem to occur here if, for example, it were discovered that for a certain animal-identification number the wrong animal was weighed. If the reset sum were updated immediately after the weighing of each animal, the old value would be lost. Then when the correct animal was weighed, the amount to feed it would be based on an incorrect reset sum. To prevent this possibility, we have written the algorithm such that the reset sum is permanently updated only after all animals have been weighed and the SAVE menu choice is selected upon exiting the COLLECT_DATA routine.

The range of the maintenance feeding for the male Long-Evans rats that we normally use is expected to be between 5 and $30 \mathrm{~g} / \mathrm{day}$. Target weights may range between 200 and $1,000 \mathrm{~g}$, with most rats stabilized at $350 \mathrm{~g}$. Rats maintained at $350-\mathrm{g}$ body weight initially require $15 \mathrm{~g} /$ day at 90 to 100 days of age; this amount declines with age. Food (Purina Rat Chow) pellets of approximately $5 \mathrm{~g}$ are selected from bags of standard feed; these are dispensed along with 1 to 4 precision $1-\mathrm{g}$ pellets, to allow a resolution of one gram in the feeding amount.

\section{RESULTS AND DISCUSSION}

The weight-maintenance algorithm was applied first to a small group $(n=6)$ of adult Long-Evans rats that were already in our animal colony and that were being maintained at $350 \mathrm{~g}$. Figure 3 shows the results of a step change in the target weight from 350 to $370 \mathrm{~g}$ at Day 10 . The group was maintained at $350 \mathrm{~g}$ for several weeks prior to the step change in target weight, but data are only shown from 10 days prior to the step change to $\mathbf{4 0}$ days

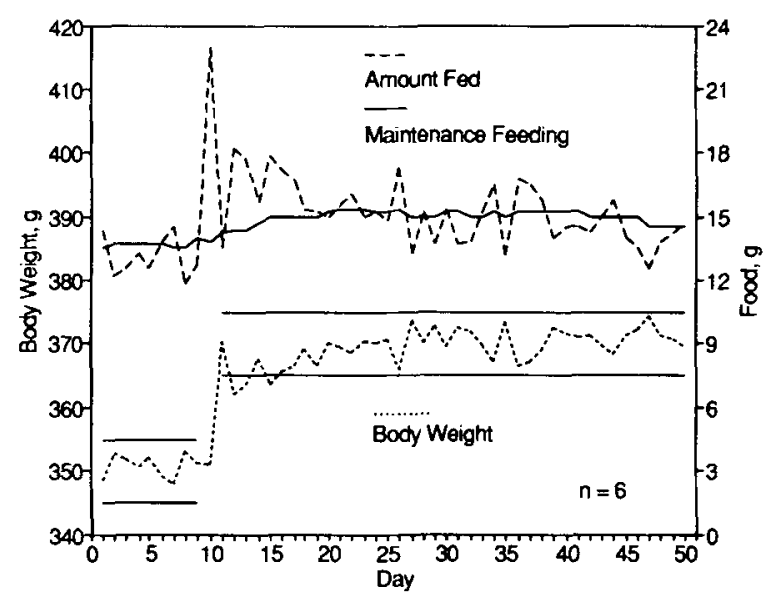

Figure 3. Body weights for a small group of adult rats from 10 days prior to a $20 \mathrm{~g}$ step change in target weight to 40 days after the change. The desired 5-g tolerance band is shown as straight lines above and below the weight data. The values for amount fed (shown) consist of the addition of two components: a maintenance-feeding term (shown), and a proportional error term (not shown). The maintenance-feeding curve shows a smooth variation because it is a summation term, whereas the amount-fed curve fuctuates more rapidly, reflecting the strong influence of the proportionsl error term. 
after the step change in the figure. The figure shows that the average body weight for the group was being maintained at $350 \mathrm{~g}$ within our desired 5-g tolerance band prior to the step change, that the maintenance feeding was relatively stable, and that the amount fed was fluctuating moderately to maintain the animals at $350 \mathrm{~g}$.

When the step change in target weight was introduced at Day 10, the proportional term responded quickly, as evidenced by the 23-g value for the amount fed on Day 10 . As desired, the maintenance feeding gradually grew larger after integrating several days of positive error terms into the reset sum. There was no overshoot in the body-weight curve and the maintenance-feeding curve shifted smoothly without oscillation, as desired. The group's mean body weight stabilized within the new tolerance band within 5 days of the setpoint change, and it remained within the band thereafter. The averaged standard error of the mean body weight was $1.1 \mathrm{~g}$.

A more typical application for the weighing station is shown in Figure 4. Here a group of young rats arrived in our animal colony and were immediately placed on the system. The mean body weight increased rapidly and entered the tolerance band within 9 days. The body weights remained within the band with the exception of 2 days. The standard error of the mean averaged over the 48-day period was $0.7 \mathrm{~g}$. Because this group of young animals already weighed approximately $325 \mathrm{~g}$ upon arrival, the 30-g limit on the amount fed was not invoked by the program; with younger, lighter animals their food would have been limited to $30 \mathrm{~g}$ until their weight exceeded about $320 \mathrm{~g}$. About half of the animals weighed less than $330 \mathrm{~g}$; therefore, for these animals, the reset-sum term was not adjusted by the program on Day 1 . This explains why the maintenance-feeding curve showed an initial small slope. After about Day 25, the curve of the amount fed began

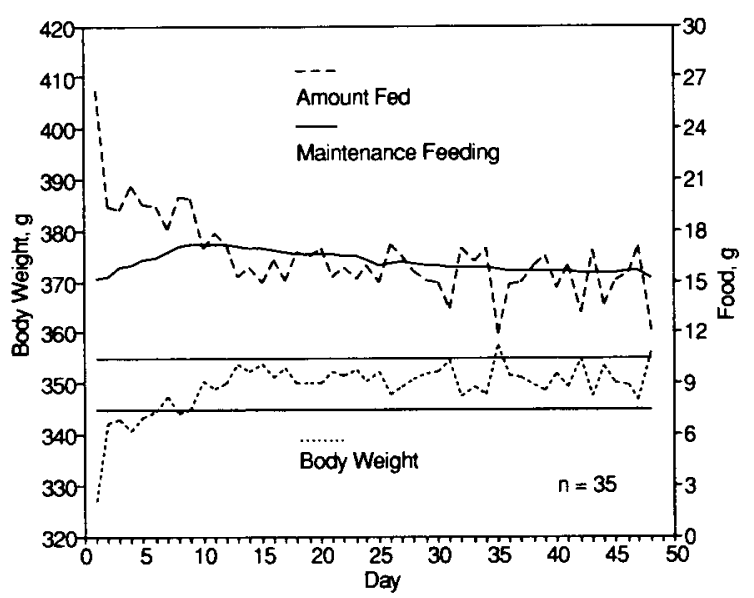

Figure 4. Body weights for newly arrived, young rats that were entered into the system with target weights of $350 \mathrm{~g}$. The mean body weight entered the tolerance band within 9 days, exhibited no overshoot, and was well maintained thereafter. The weight-maintenance algorithm adjusted the maintenance feeding gradually and without oscillation, as desired. to fluctuate above and below the maintenance-feeding curve. This indicates that the maintenance feeding has been adjusted to the daily caloric need of the rat for the given target weight.

In addition to the improved control of body weight, the system also provides advantages of ease of use by animalcare personnel and facilitation of recordkeeping for purposes of quality assurance. This system was readily accepted by the animal-care staff in our laboratory and was found to reduce their work load by automating recordkeeping and calculation of amounts to feed. Fewer errors of feeding amounts are now committed, and weekend staff can provide correct amounts of food to the rats as well. Since records of weight and amount fed are generated, transferred to a centralized minicomputer, and stored, a database is generated for monitoring animal health and response to treatment.

The software is readily amenable to calculation of dosages of drugs and toxicants. However, in our case, maintaining weights at a constant value for an entire experiment removes the need to calculate dose volumes for individual animals. In other studies, in which body weights may be maintained at different target levels, calculation of individual dosages would be more beneficial.

\section{Availability}

This Lotus worksheet, including macros, two sample balance setting files for Measure, and a batch file called MERGE.BAT are available free of charge from the authors. Send an MS-DOS-formatted 5.25-in. doublesided, double-density floppy disk and a diskette mailer to J. S. Ali, USEPA, MD-74B, Research Triangle Park, NC 27711.

\section{REFERENCES}

GAD, S. C. (1982). A neuromuscular screen for use in industrial toxicology. Journal of Toxicology \& Environmental Health, 9, 691-704.

HiNe LiNE, P. N. (1986). Editorial: The relationships between subject and experimenter. Joumal of the Experimental Analysis of Behavior, 45, 123-132.

Holehan, A. M., \&erry, B. J. (1986). The experimental manipulation of aging by diet. Biological Reviews, 61, 329-386.

Hurwitz, H. M. B., \& DAvis, H. (1983). Depriving rats of food: A reappraisal of two techniques. Journal of the Experimental Analysis of Behavior, 40, 211-213.

Kaplan, M., Campbell, S. L., Johnson, L., PapaMichael, A., Sparer, R., WeInBaum, M. (1959). Growth of body weight and manipulation of food motivation. Science, 129, 1673-1674.

Moser, V. C., McCormick, J. P., Creason, J. P., MacPhail, R. C. (1988). Comparison of chlordimeform and carbaryl using a functional observational battery. Fundamental \& Applied Toxicology, 11, 189-206.

PAgAN, J. D. (1989). Reevaluating nutrient needs. Equus, 141, 113$116,128$.

Yu, B. P., Masoro, E. J., Murata, I., Bertrand, H. A., \& Lynd, F. T. (1982). Lifespan study of SPF Fisher 344 male rats fed ad libitum or restricted diets: Longevity, growth, lean body mass and disease. Journal of Gerontology, 37, 130-141.

ZBINDEN, G. (1988). Reduction and replacement of laboratory animals in toxicological testing and research. Biomedical \& Environmental Science, 1, 90-100.

(Manuscript received February 11, 1991; revision accepted for publication July 17,1991 .) 